\title{
A new production prediction model for multistage fractured horizontal well in tight oil reservoirs
}

\author{
Kui Zhao ${ }^{1,2}$, Peng $\mathrm{Du}^{1 \oplus *}$ \\ ${ }^{1}$ Qingdao Jari Industry Control Technology, Qingdao 266000, P. R. China \\ ${ }^{2}$ College of Information Science and Engineering, Ocean University of China, Qingdao 266100, P. R. China
}

Keywords:

Production prediction

type curves

transient analysis

tight formation

Cited as:

Zhao, K., Du, P. A new production prediction model for multistage fractured horizontal well in tight oil reservoirs. Advances in Geo-Energy Research, 2020, 4(2): 152-161,

doi: $10.26804 /$ ager.2020.02.04.

\begin{abstract}
:
To better evaluate the production performance of tight oil reservoirs, it is urgent to solve the multistage fractured horizontal well production enigma. It is paramount to develop new models to analyze the well performance for tight oil reservoirs. In this paper, a new production prediction model of multistage fractured horizontal well in tight oil reservoir was established. In this model, unsteady transfer flow between fracture and matrix was considered. This model was solved by using Laplace transform method, line source function and Stehfest method comprehensively. The production prediction type curves including pressure transient analysis curves and rate transient analysis curves were then obtained. According to these type curves, eight flow regimes were obtained as early wellbore storage period, skin factor period, bi-linear flow regime, linear flow regime, first radial flow regime, transition flow regime, transfer flow regime and later radial flow regime. In the end, a field case history matching result was given and four key parameters' effect on tight formation well production was analyzed. This research is of both theoretical significance and practical value for tight oil development.
\end{abstract}

\section{Introduction}

Unconventional resource development has became a hot issue recently in the world and is gradually becoming a key component in the world's energy supply (Wang et al., 2017; Zhang et al., 2018; Cai et al., 2019; Yu et al., 2019). Due to extremely low permeability ranging from $10^{-2}$ to $10^{-6} \mathrm{mD}$ (Cipolla et al., 2009), horizontal well drilling and multistage hydraulic fracturing have been proven to be an integral tool for tight oil/gas production. The economic feasibility of tight reservoirs has a strong relationship with the fracture system permeability near the wellbore (Mayerhofer et al., 2010; Brown et al., 2011; Xie et al., 2015).

When developing a tight oil/gas reservoir, well production performance should be predicted and thus a reasonable plan can be drawn up. Now, many research models to predict the multistage fractured horizontal well (MsFHW) performance were proposed which are worthy to be reviewed. Larsen and Hegre (1994) showed the transient pressure solution for multiple transverse fractured well. Some flow regimes were divided without considering boundary effect. Zerzar and Bettam
(2003) also presented an analytical model for fractured horizontal wells in anisotropic closed or semi-infinite, homogenous or naturally fractured systems. Three assumptions including uniform flux, infinite conductivity and finite conductivity models were considered (Larsen and Hegre, 1994). Coupling the numerical fracture with an analytical reservoir model, AlKobaisi et al. (2004) presented a hybrid numerical/analytical model for pressure transient response of a finite conductivity fracture intercepted by a horizontal well. The work can be applied to develop multiply fractured horizontal well models by superposition. "Linear flow model" was proposed for pressure transient analysis (PTA) (Ozkan et al., 2004; Brown et al., 2011; Ozcan et al., 2014). This is a powerful model which is fit for ultra-low permeability reservoir development. After that, Stalgorova and Mattar (2012a, 2012b) extended the "trilinear flow model" to "five region model" in which the formation is partially improved by the fracturing between two adjacent hydraulic fractures. Then the semi-analytical methods were presented in which the complex shape fracture was considered. In these models, the hydraulic fracture can be

${ }^{*}$ Corresponding author.

E-mail address: zhaokui716@163.com (K. Zhao); dupengjari@163.com (P. Du). 
non-planar with finite conductivity. Sensitive parameters were analyzed carefully such as fracture spacing, wellbore storage, and fracture conductivity (Luo et al., 2014; Wang et al., 2014; Luo and Tang, 2015).

Recently, some complex geometry shape models were also established to satisfy the complex geometry after fracturing in tight/oil reservoir. Jiang et al. (2014) and Zhao et al. (2015) established the circle composite reservoir model. This model assumes that there are two regions in reservoir. The inner region and outer region have different parameters and coupled using the interface conditions. The source function was used to solve the models. PTA and rate transient analysis (RTA) were analyzed in detail (Jiang et al., 2014; Zhao et al., 2014, 2015). Similarly, Ketineni and Ertekin (2012) established the composite model for multi-stage hydraulically fractured horizontal well in a naturally fractured reservoir considering the stimulated reservoir volume (SRV). They take the SRV as a "rubble zone" and they assumed a pseudo-steady model to describe the transfer of the fluid from matrix to fracture. They didn't give the exact pressure transient analysis results.

In this paper, the multistage fractured horizontal well PTA and RTA models were established. In matrix system, the unsteady flow was considered and the sphere block matrix was used. In section 2, the physical model and mathematical model were established. In section 3, the solution was showed in detail. In section 4, we verified the model using some previous results. At the same time, different flow regimes were divided according to pressure transient and rate transient curves. In the last section, the sensitivity analyses for four different parameters were given to make us a better understanding of the performance of MsFHW.

\section{Model construction}

In this section, we discuss the physical and mathematical models for understanding MsFHW performance.

\subsection{Physical model}

The schematic diagram for MsFHW is shown in Fig. 1. The formation is a dual porosity media including matrix, natural fractures shown in Fig. 2. The model assumes: (1) the reservoir is horizontal with uniform thickness of $h$ and original pressure $p_{i}$; (2) the horizontal permeability is $k_{f h}$, the vertical permeability is $k_{f v}$, the compressibility is $C_{t f}$, the porosity is $\phi_{f}$ for the fracture system; for the matrix, the permeability is $k_{m}$, the compressibility is $C_{t m}$, the porosity is $\phi_{m}$; (3) the influence of gravity and capillary forces on fluid flow in both regions is ignored; (4) the wellbore storage effect and formation damage are taken into account. The well produces at a constant rate is $q_{s c}$; and (5) spherical matrix blocks are considered with the uniform radius $R$.

\subsection{Mathematical model}

For the matrix system, unsteady flow is considered. The flow equation can be expressed as follows:

In the spherical coordinates system:

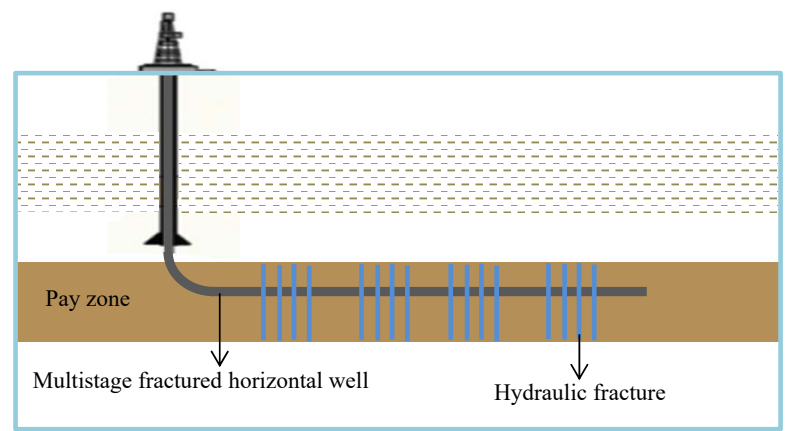

Fig. 1. Multistage fractured horizontal well.

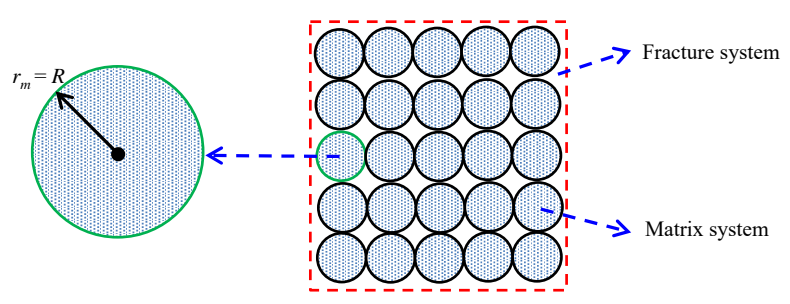

Fig. 2. Schematic of fracture and matrix system (after Yuan et al., 2018).

$$
\frac{1}{r_{m}^{2}} \frac{\partial}{\partial r_{m}}\left(r_{m}^{2} \frac{\partial p_{m}}{\partial r_{m}}\right)=\frac{\phi_{m} \mu C_{t m}}{k_{m}} \frac{\partial p_{m}}{\partial t} \quad\left(0 \leq r_{m} \leq R\right)
$$

Initial condition:

$$
p_{m}\left(r_{m}, 0\right)=p_{i}
$$

Inner boundary condition:

$$
\left.\frac{\partial p_{m}}{\partial r_{m}}\right|_{r_{m}=0}=0
$$

Outer boundary condition:

$$
\left.p_{m}\right|_{r_{m}=R}=p_{f}
$$

For the fracture system, the flow equation in the inner region is as follows with cylindrical coordinates system:

$$
\begin{aligned}
& \frac{1}{r} \frac{\partial}{\partial r}\left(\frac{k_{f h}}{\mu} r \frac{\partial p_{f}}{\partial r}\right)+\frac{k_{f h}}{\mu} \frac{1}{r^{2}} \frac{\partial^{2} p_{f}}{\partial \theta^{2}}+\frac{k_{f v}}{\mu} \frac{\partial^{2} p_{f}}{\partial z^{2}}-\left.\frac{3}{R} \frac{k_{m}}{\mu} \frac{\partial p_{m}}{\partial r_{m}}\right|_{r_{m}=R} \\
& =\phi_{f} C_{t f} \frac{\partial p_{f}}{\partial t}
\end{aligned}
$$

With Eqs. (1)-(5), the flow equation can be expressed as follows (Details are presented in Appendix A and Appendix B):

$$
\begin{aligned}
& \frac{1}{r_{D}} \frac{\partial}{\partial r}\left(r_{D} \frac{\partial \bar{p}_{f 1 D}}{\partial r_{D}}\right)+\frac{1}{r_{D}^{2}} \frac{\partial^{2} \bar{p}_{f D}}{\partial \theta^{2}}+\frac{\partial^{2} \bar{p}_{f D}}{\partial z_{D}^{2}}=f \bar{p}_{f D} \\
& f=\omega u+3 \lambda\left(\sqrt{\frac{1-\omega}{\lambda}} u \operatorname{coth} \sqrt{\left.\frac{1-\omega}{\lambda} u-1\right)}\right.
\end{aligned}
$$


Inner boundary conditions:

$$
\left.\lim _{\varepsilon_{D} \rightarrow 0} r_{D} \frac{\partial \bar{p}_{f D 01}}{\partial r_{D}}\right|_{r_{D}=p_{f D}}=-\frac{\overline{\tilde{q}}_{L}}{q_{s c}}
$$

Upper and lower boundary condition:

$$
\frac{\partial \bar{p}_{f D}}{\partial z_{D}}=0, z_{D}=0 \text { or } h_{D}
$$

Outer boundary condition:

$$
\bar{p}_{f D}=0, r_{D}=\infty
$$

Initial condition:

$$
\bar{p}_{f D}=0, t_{D}=0
$$

\section{Analytical solution}

Eqs. (6)-(10) form the basic model for the composite reservoir with a line source. There are two methods to obtain pressure solution for the model proposed above. One is using point function method (Jiang et al., 2014). This method is based on point function solution. Then the line source can be obtained by point function solution integral. The other one is to obtain line source solution directly according to characteristics of Dirac delta function and Bessel's function (Zhao et al., 2014). Both methods can solve this model effectively. The pressure distribution for a line source is:

$$
\bar{p}_{D}=\frac{\overline{\tilde{q}}_{L}}{q_{s c}} K_{0}\left(\sqrt{f} R_{D}\right)
$$

where $R_{D}$ is the distance between the line source and pressure observation point. Now, the continuous point sources solution has been derived. Then, using line source function superposition principle (Jiang et al., 2014; Zhao et al., 2014), the wellbore pressure $\bar{p}_{w D}$ in Laplace space can be obtained. To consider the finite conductivity fracture in transient pressure or rate analysis, the solution method which is proposed by Cinco et al. (1978) is used in our model.

Using Duhamel's principle (Van Everdingen and Hurst, 1949), the bottomhole pressure solution is obtained considering well storage effect and skin effect:

$$
\bar{p}_{w D}\left(S, C_{D}\right)=\frac{u \bar{p}_{w D}+S}{u+C_{D} u^{2}\left[u \bar{p}_{w D}+S\right]}
$$

The dimensionless well production rate at a constant wellbore pressure can be defined as follows (Van Everdingen and Hurst, 1949):

$$
\bar{q}_{D}=\frac{1}{u^{2} \bar{p}_{w D}}
$$

\section{Type curves}

To obtain the dimensionless wellbore pressure at a constant dimensionless production rate or production at a constant wellbore pressure in real space, the Stehfest method can be used to transform the Laplace space value into the real space (Cinco et al., 1978; Jiang et al., 2014). The code of the frame-

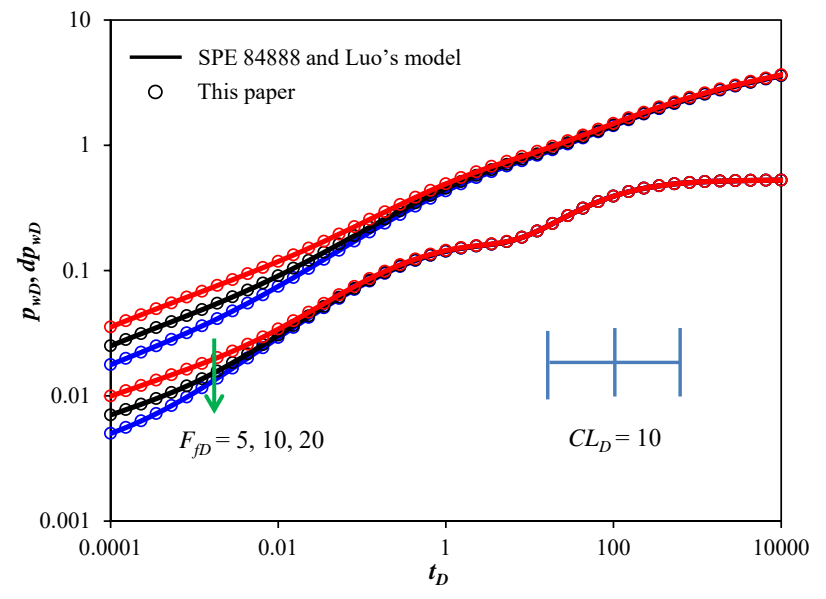

Fig. 3. Comparison of pressure and pressure derivative for multistage fractured horizontal well by 3 hydraulic fractures.

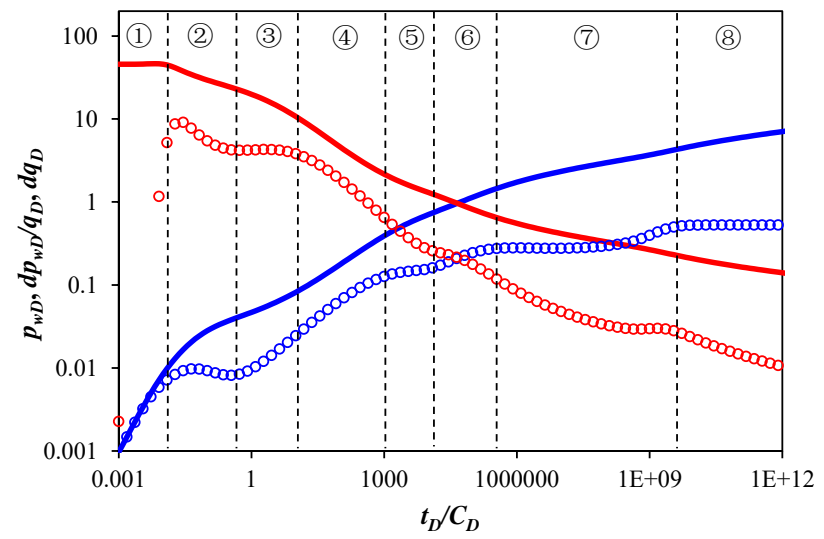

Fig. 4. Type curves for MsFHW. Basic parameters: $M=3 ; D L_{D}=10 ; L_{f}=$ $50 ; \omega=0.015 ; \lambda=0.00001 ; C_{D}=0.00001 ; S=0.02 ; R_{f D}=20$.

work was programed by Matlab 2013a. Then type curves can be analyzed.

\subsection{Model verification}

In this model, if some parameters are set to satisfy some conditions, the new model proposed in this paper can be converted into some other models. If we set $f=u$, this new model can be simplified as single-porosity model in a homogenous reservoir, this simplified model is similar with the model proposed by Zerzar and Bettam (2003) and Luo et al. (2014). Fig. 3 shows the bottomhole pressure curves. Seen from Fig. 3, this new model can match with the existing model. This new model is reliable. Also, in our model, the parameters are set the same as Yuan et al. (2018) (See Fig. 3 in their paper), the results are matched well.

\subsection{Type curves}

The type curves are demonstrated in Fig. 4, and the following eight flow regimes are divided based on the type curves. 
Table 1. Basic parameters for multistage fractured horizontal well in a tight oil reservoir.

\begin{tabular}{lll}
\hline Parameters & Unit & Value \\
\hline Formation thickness, $h$ & $\mathrm{~m}$ & 7 \\
Wellbore radius, $r_{w}$ & $\mathrm{~m}$ & 0.1 \\
Hydraulic-fracture half length, $L_{f}$ & $\mathrm{~m}$ & 100 \\
Viscosity, $\mu$ & $\mathrm{Pa} \cdot \mathrm{s}$ & $2.6 \times 10^{-3}$ \\
Skin factor, $S$ & $\mathrm{dimensionless}$ & 0.005 \\
Wellbore storage coefficient, $C$ & $\mathrm{~m}^{3} / \mathrm{Pa}$ & $0.01 \times 10^{-6}$ \\
Matrix radius, $R$ & $\mathrm{~m}$ & 50 \\
Fracture permeability, $k_{f h}$ & $\mathrm{~m} 2$ & $2 \times 10^{-15}$ \\
Matrix permeability, $k_{m}$ & $\mathrm{~m}{ }^{2}$ & $0.0001 \times 10^{-15}$ \\
Fracture porosity, $\phi_{f}$ & $\mathrm{fraction}$ & 0.001 \\
Matrix porosity, $\phi_{m}$ & $\mathrm{fraction}$ & 0.05 \\
Fracture compressibility, $C_{t f}$ & $1 / \mathrm{Pa}$ & $0.0006 \times 10^{-6}$ \\
Matrix compressibility, $C_{t m}$ & $1 / \mathrm{Pa}$ & $0.0008 \times 10^{-6}$ \\
Hydraulic fracture conductivity, $R_{f}$ & $\mathrm{mD} \cdot \mathrm{cm}$ & $4 \times 10^{5}$ \\
Bottom hole flowing pressure, $P_{w}$ & $\mathrm{~Pa}$ & $6 \times 10^{6}$ \\
Hydraulic fracture number, $N$ & integer & 12 \\
Fracture spacing, $D L$ & $\mathrm{~m}$ & 100 \\
Production pressure difference, $\Delta P$ & $\mathrm{MPa}$ & 7 \\
\hline
\end{tabular}

Regime (1): The early wellbore storage period. The slope is 1 in both the pressure and pressure derivative curve in log-log coordinates.

Regime (2): The skin factor period. This period is between wellbore storage and the early bilinear flow.

Regime (3): Bi-linear flow regime. During this regime, the gas/oil in fracture will flow into the wellbore perpendicular to the hydraulic fracture. Both the pressure and pressure derivative curves show a straight line with $1 / 4$ slope.

Regime (4): Linear flow regime. During this regime, the gas/oil in fracture will flow into the wellbore perpendicular to the hydraulic fracture. Both the pressure and pressure derivative curves show a straight line with $1 / 2$ slope.

Regime (5): First radial flow regime. Following regime (4), if the distance of two adjacent hydraulic fractures is long enough, this regime will happen. The value in pressure derivative curves is constant which equal to $0.5 / \mathrm{M}$.

Regime (6): Transition flow regime. This regime happens between regime (5) and regime (1). This regime may disappear when $\lambda$ is big.

Regime (7): Transfer flow regime. In this regime, the oil/gas will flow from matrix to fracture because the pressure in fracture decreases largely and the flow from matrix to fracture becomes an important supplement flowing into the fracture. A concave appears in pressure derivative curves. It is a typical characteristic for dual porosity media.

Regime (8): Later radial flow regime. Following regime (1), this regime will happen when the oil/gas flows from fracture into wellbore and from matrix to fracture get to a balance state. The value in pressure derivative curves is constant which equal to 0.5 .

\section{Field case study and effect of relevant parameters}

\subsection{Field case study}

F154 block is a fault-lithology tight oil reservoir located at Gaoqing City, Shandong Province, China. The reservoir is controlled by faults on the north and south. The objective interval belongs to the third member of Shahejie Formation whose sandstone thickness is $0-13 \mathrm{~m}$ and the top depth between $2769-2830 \mathrm{~m}$. Because of the ultra-low permeability, MsFHW was used to develop this block. Well-P1 is a typical well in this block and has 12 hydraulic fractures. The basic parameters for Well-P1 are shown in Table 1. Fig. 5 shows the history result, the matching result is good enough which indicates the practical value of the model in this paper.

\subsection{Effect of relevant parameters}

To better known the effect of different parameters on tight oil/gas production performance, in this section, four key parameters were analyzed. The basic reservoir parameters used are from Well-P1.

\subsubsection{Effect of natural fracture permeability}

Fig. 6 and Fig. 7 show the natural fracture permeability effect on transient pressure curves and oil accumulative production. It can be seen from Fig. 6 that the natural fracture permeability mainly affects the regimes from linear regime to transfer flow regimes. High natural fracture permeability leads 
to a narrow linear flow regime. The reason is that high natural fracture permeability makes the pressure spreads quickly and thus the linear is not obvious. From Fig. 7, we can see that high permeability increases the well accumulative production. When developing tight oil/gas reservoirs, if the formation permeability is ultra-low, it's a better way to fracture the formation and thus high flow capacity can be created around

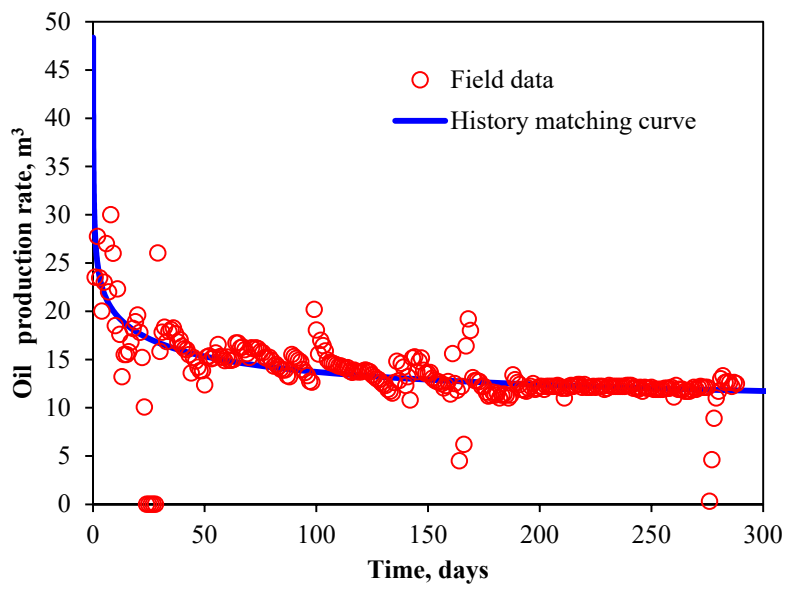

Fig. 5. History matching between the model result and real production data.

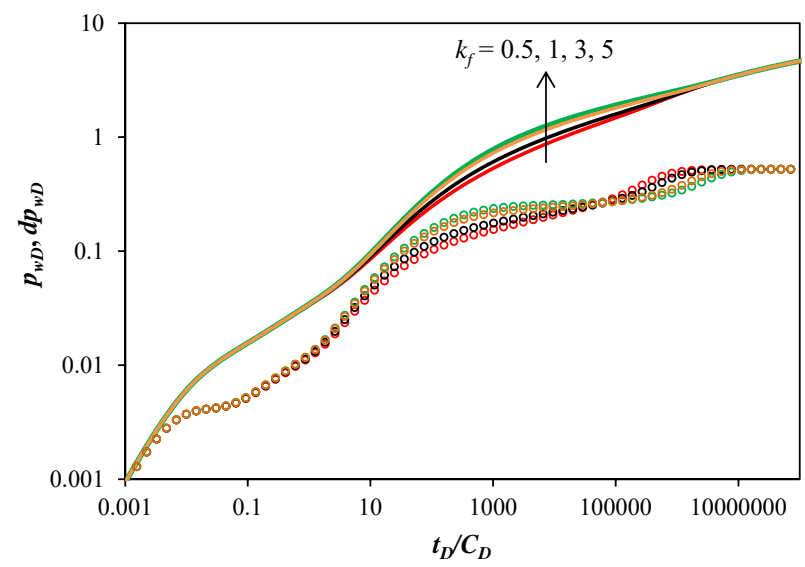

Fig. 6. Effect of natural fracture permeability on transient pressure curves.

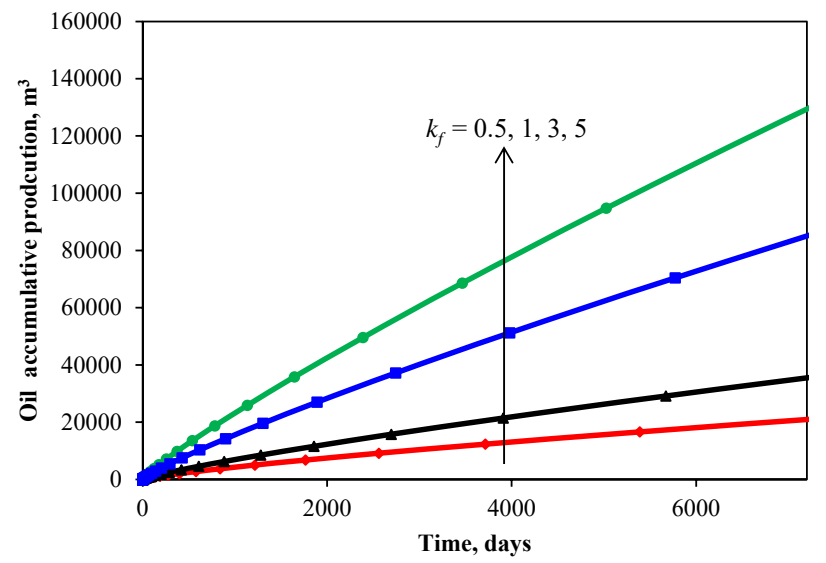

Fig. 7. Oil accumulative production in 20 years predicted with different natural fracture permeability. wellbore. That is why the high flow capacity needs to be created. It is a key technique to make "high flow region" which also is called SRV (Brown et al., 2011; Stalgorova et al., 2012a) in tight oil/gas development.

\subsubsection{Effect of matrix permeability}

Fig. 8 and Fig. 9 show the matrix permeability effect on transient pressure curves and oil accumulative production. As can be seen from Fig. 8, the matrix permeability affects the regimes after linear flow regime. High matrix permeability leads to the transfer flow regime early. The reason is that high matrix permeability makes it easy to flow between the matrix and fracture and thus the pressure drop is smaller compared with low matrix permeability. From Fig. 9, we can see that high matrix permeability leads to high oil accumulative production. Therefore, when a tight oil/gas has a very low permeability, it is necessary to fracture the formation to achieve high accumulative production.

\subsubsection{Effect of matrix size}

Fig. 10 and Fig. 11 show the matrix size effect on transient pressure curves and oil accumulative production. As can be seen from Fig. 10, the matrix size affects the regimes from

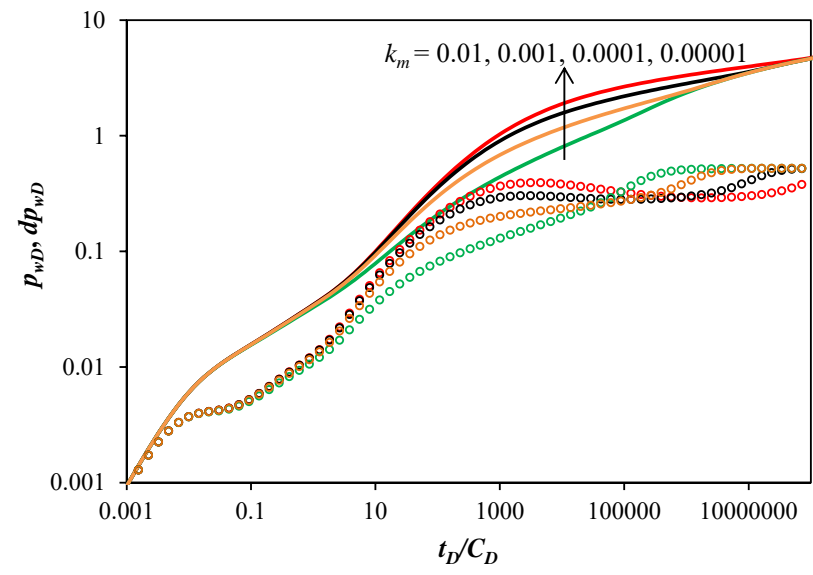

Fig. 8. Effect of matrix permeability on transient pressure curves.

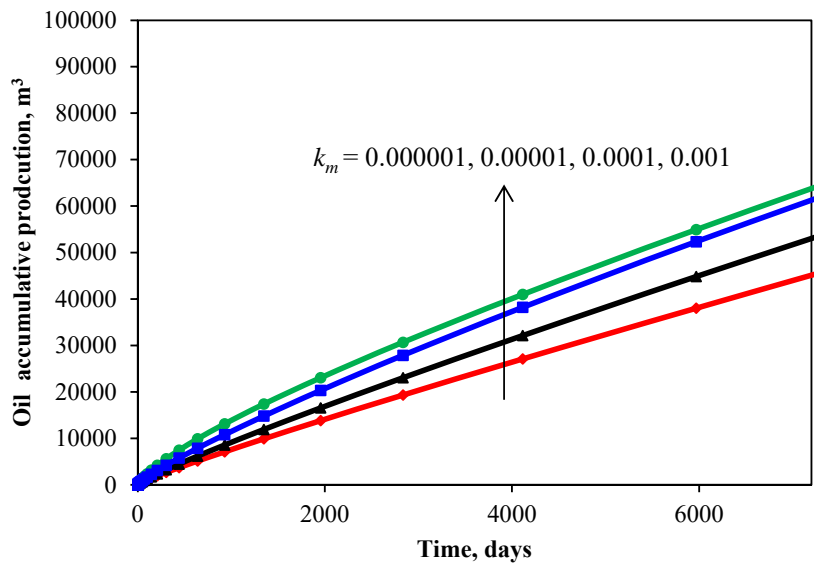

Fig. 9. Oil accumulative production in 20 years predicted with different matrix permeability. 


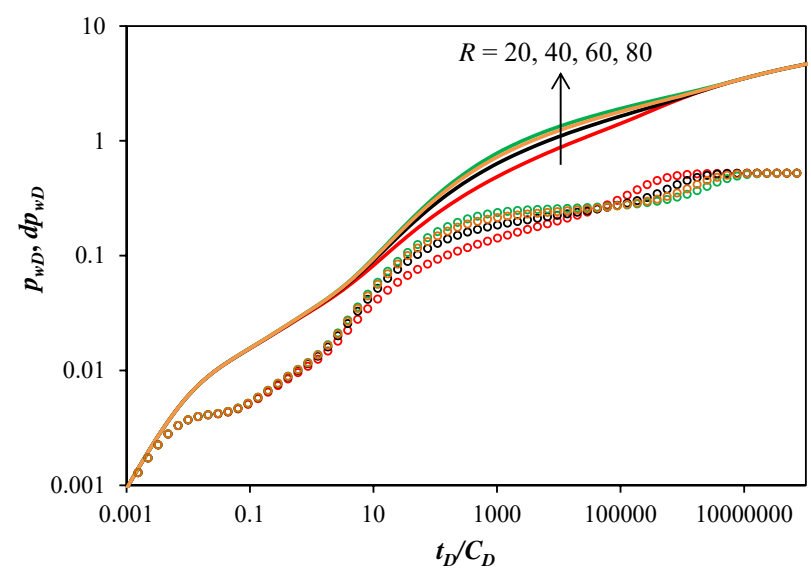

Fig. 10. Effect of matrix size on transient pressure curves.

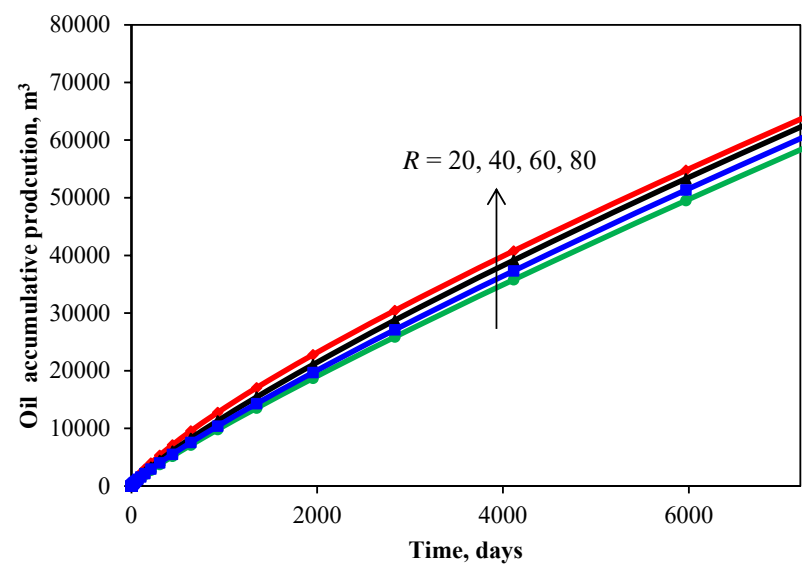

Fig. 11. Oil accumulative production in 20 years predicted with different matrix size.

linear flow regime to transfer flow regime. Big matrix size leads to a high pressure drop. The reason is that big matrix size makes the contact surface of matrix and fracture smaller compared with small matrix size. So the inter-porosity flow amount between matrix and fracture is smaller. From Fig. 11, we can see that small matrix size leads to low oil accumulative production while the effect is relatively smaller than that natural fracture permeability and matrix permeability.

\subsubsection{Effect of fracture spacing}

Fig. 12 and Fig. 13 show the fracture spacing effect on transient pressure curves and oil accumulative production. As can be seen from Fig. 12, the fracture spacing affects the regimes from linear flow regime to transfer flow regime. The pressure drop is relatively small when the fracture spacing is big. The reason is that big fracture spacing can make the hydraulic fracture have a big drainage oil area and thus the flow resistance is smaller around the wellbore. Also, this can lead to high oil accumulative production seen from Fig. 13.

\section{Conclusions}

The main purpose of this work is to present the model

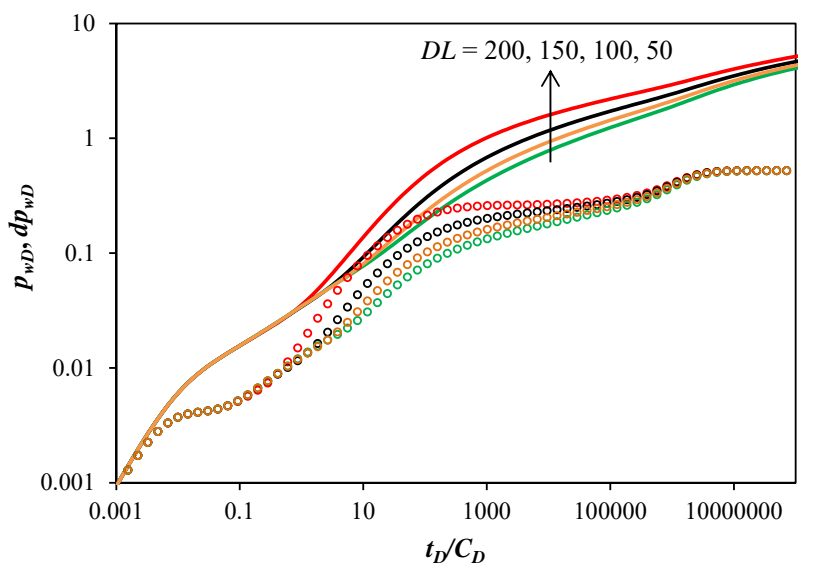

Fig. 12. Effect of fracture spacing on transient pressure curves.

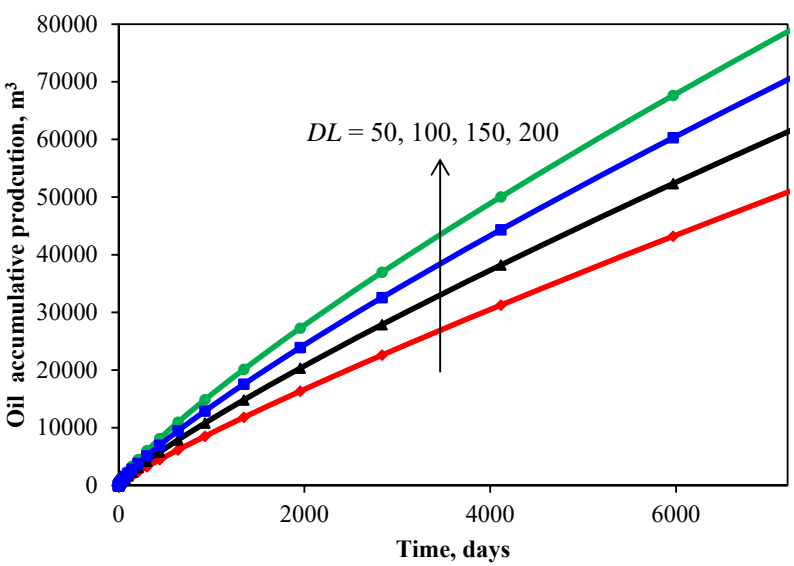

Fig. 13. Oil accumulative production in 20 years predicted with different fracture spacing.

and results of an extended study on the performance analysis of multistage fractured horizontal wells. The motivations for the research subject of this study came from the fact that the complexity of hydraulically fractured wells is always greatly affected by hydraulic fracturing. In this paper, with the semi-analytical method, the performance analysis model was established considering unsteady transfer flow between matrix and fracture. Sensitivity analyses over four uncertain parameters were given. Based on the results presented, the following conclusions are obtained:

1) The mathematical model was derived for performance analysis of multistage fractured wells. Eight possible flow regimes exists based on pressure transient analysis and rate transient analysis which are the early wellbore storage period, the skin factor period, bi-linear flow regime, linear flow regime, first radial flow regime, transition flow regime, transfer flow regime, and later radial flow regime.

2) The model can be used for real data history matching. The parameters history matching results for Well-P1 indicates this model is a good tool for MsFHW production prediction.

3) Natural fracture permeability and fracture spacing has great effect on well productivity. A higher natural fracture 
permeability and bigger fracture spacing always leads to higher gas production rate because they either increase the flow capacity in reservoir or increase the drainage area. Matrix permeability and matrix size also have effects on well productivity. A higher matrix permeability and small matrix size always lead to higher gas production rate because they increase the flow mass between matrix and fracture.

\section{Nomenclature}

$k=$ Permeability, $\mathrm{m}^{2}$

$\mu=$ Fluid viscosity, $\mathrm{Pa} \cdot \mathrm{s}$

$\eta=$ Diffusivity ratio, fraction

$u=$ Laplace space variable with respect to $t_{D}$

$h=$ Reservoir thickness, $\mathrm{m}$

$L=$ Reference length, $\mathrm{m}$

$K_{k}(x)=$ The modified Bessel function of second kind

$q_{s c}=$ Production rate, $\mathrm{m}^{3} / \mathrm{s}$

$C=$ Wellbore storage, $\mathrm{m}^{3} / \mathrm{Pa}$

$S=$ Skin factor, dimensionless

$L_{f}=$ Fracture half length, $\mathrm{m}$

$t=$ Time, $\mathrm{s}$

$N=$ Facture number

$D L=$ Fracture spacing, $\mathrm{m}$

$r, \theta, z=$ Directional coordinates

$\phi=$ Porosity, fraction

$C_{t}=$ Total compressibility, $\mathrm{Pa}^{-1}$

$p=$ Pressure, $\mathrm{Pa}$

$\omega=$ Storability coefficient

$\lambda=$ Inter-porosity coefficient

$\tilde{q}=$ Point source, $\mathrm{m}^{3} / \mathrm{s}$

$R=$ Matrix radius, $\mathrm{m}$

$r_{m}=$ Distance to the matrix center, $\mathrm{m}$

$R_{f}=$ Hydraulic fracture conductivity, $\mathrm{mD} \cdot \mathrm{cm}$

\section{Subscripts and superscripts}

$D=$ Dimensionless

$i=$ Initial

$h=$ Horizontal direction

$v=$ Vertical direction

$f=$ Fracture

$-=$ Laplace domain

$m=$ Matrix

\section{Acknowledgement}

This work is supported by Qingdao Shinan Zone Science \& Technology Plan (2018-3-005XXGK18807).

\section{Conflict of interest}

The authors declare no competing interest.

Open Access This article, published at Ausasia Science and Technology Press on behalf of the Division of Porous Flow, Hubei Province Society of Rock Mechanics and Engineering, is distributed under the terms and conditions of the Creative Commons Attribution (CC BY-NC-ND) license, which permits unrestricted use, distribution, and reproduction in any medium, provided the original work is properly cited.

\section{References}

Al-Kobaisi, M., Ozkan, E., Kazemi, H. A hybrid numericalanalytical model of finite-conductivity vertical fractures intercepted by a horizontal well. Paper SPE 92040 Presented at SPE International Petroleum Conference in Mexico, Puebla Pue, Mexico, 7-9 November, 2004.

Brown, M., Ozkan, E., Raghavan, R., et al. Practical solutions for pressure-transient responses of fractured horizontal wells in unconventional shale reservoirs. SPE Reserv. Eval. Eng. 2011, 14(6): 663-676.

Cai, J., Lin, D., Singh, H., et al. A simple permeability model for shale gas and key insights on relative importance of various transport mechanisms. Fuel 2019, 252: 210-219.

Cinco, L., Samaniego, V., Dominguez, A. Transient pressure behavior for a well with a finite-conductivity vertical fracture. Soc. Pet. Eng. J. 1978, 18(4): 253-264.

Cipolla, C.L., Lolon, E., Mayerhofer, M.J. Reservoir modeling and production evaluation in shale-gas reservoirs. Paper IPTC 13185 Presented at International Petroleum Technology Conference, Doha, Qatar, 7-9 December, 2009.

Jiang, R., Xu, J., Sun, Z., et al. Rate transient analysis for multistage fractured horizontal well in tight oil reservoirs considering stimulated reservoir volume. Math. Probl. Eng. 2014, 2014: 489015.

Ketineni, S.P., Ertekin, T. Analysis of production decline characteristics of a multistage hydraulically fractured horizontal well in a naturally fractured reservoir. Paper SPE 161016 Presented at SPE Eastern Regional Meeting, Lexington, Kentucky, USA, 3-5 October, 2012.

Larsen, L., Hegre, T.M. Pressure transient analysis of multifractured horizontal wells. Paper SPE 28389 Presented at SPE Annual Technical Conference and Exhibition, New Orleans, Louisiana, 25-28 September, 1994.

Luo, W., Tang, C. Pressure-transient analysis of multiwing fractures connected to a vertical wellbore. SPE J. 2015, 20(2): 360-367.

Luo, W., Tang, C., Wang, X. Pressure transient analysis of a horizontal well intercepted by multiple non-planar vertical fractures. J. Pet. Sci. Eng. 2014, 124: 232-242.

Mayerhofer, M.J., Lolon, E., Warpinski, N.R., et al. What is stimulated reservoir volume? SPE Prod. Oper. 2010, 25(1): 89-98.

Ozcan, O., Sarak, H., Ozkan, E., et al. A trilinear flow model for a fractured horizontal well in a fractal unconventional reservoir. Paper SPE 170971 Presented at SPE Annual Technical Conference and Exhibition, Amsterdam, The Netherlands, 27-29 October, 2014.

Ozkan, E., Brown, M.L., Raghavan, R.S., et al. Comparison of fractured horizontal-well performance in conventional and unconventional reservoirs. Paper SPE 121290 Presented at SPE Western Regional Meeting, San Jose, California, 24-26 March, 2004.

Stalgorova, E., Mattar, L. Analytical model for history matching and forecasting production in multifrac composite 
systems. Paper SPE 162516 Presented at SPE Canadian Unconventional Resources Conference, Calgary, Alberta, Canada, 30 October-1 November, 2012a.

Stalgorova, E., Mattar, L. Practical analytical model to simulate production of horizontal wells with branch fractures. Paper SPE 162515 Presented SPE Canadian Unconventional Resources Conference, Calgary, Alberta, Canada, 30 October-1 November, 2012b.

Van Everdingen, A.F., Hurst, W. The application of the Laplace transformation to flow problems in reservoirs. J. Pet. Technol. 1949, 1(12): 305-324.

Wang, W., Zheng, D., Sheng, G., et al. A review of stimulated reservoir volume characterization for multiple fractured horizontal well in unconventional reservoirs. Adv. GeoEnergy Res. 2017, 1(1): 54-63.

Wang, X., Luo, W., Hou, X., et al. Pressure transient analysis of multi-stage fractured horizontal wells in boxed reservoirs. Pet. Explor. Dev. 2014, 41(1): 82-87.

Xie, J., Yang, C., Gupta, N., et al. Integration of shalegas-production data and microseismic for fracture and reservoir properties with the fast marching method. SPE J. 2015, 20(2): 347-359.

Yuan, J., Jiang, R., Zhang, W. The workflow to analyze hydraulic fracture effect on hydraulic fractured horizontal well production in composite formation system. Adv. Geo-Energy Res. 2018, 2(3): 319-342.

Yu, Y., Chen, Z., Xu, J. A simulation-based method to determine the coefficient of hyperbolic decline curve for tight oil production. Adv. Geo-Energy Res. 2019, 3(4): 375-380.

Zerzar, A., Bettam, Y. Interpretation of multiple hydraulically fractured horizontal wells in closed systems. Paper SPE 84888 Presented at SPE International Improved Oil Recovery Conference in Asia Pacific, Kuala Lumpur, Malaysia, 20-21 October, 2003.

Zhang, Q., Su, Y., Zhao, H., et al. Analytic evaluation method of fractal effective stimulated reservoir volume for fractured wells in unconventional gas reservoirs. Fractals 2018, 26(6): 1850097.

Zhao, Y., Zhang, L., Liu, Y., et al. Transient pressure analysis of fractured well in bi-zonal gas reservoirs. J. Hydrol. 2015, 524: 89-99.

Zhao, Y., Zhang, L., Luo, J., et al. Performance of fractured horizontal well with stimulated reservoir volume in unconventional gas reservoir. J. Hydrol. 2014, 512: 447456. 


\section{Appendix A}

Dimensionless variables:

$$
\begin{gathered}
p_{D}=\frac{2 \pi k_{f h} h\left(p_{i}-p\right)}{q_{s c} \mu} \\
C_{D}=\frac{C}{2 \pi\left(\phi C_{t}\right)_{t+m} h L^{2}} \\
t_{D}=\frac{k_{f h} t}{\left(\phi C_{t}\right)_{m+f} \mu L^{2}} \\
z_{D}=\frac{z}{L} \sqrt{\frac{k_{f h}}{k_{f v}}} \\
r_{D}=\frac{r}{L} \\
h_{1 D}=\frac{h}{L} \sqrt{\frac{k_{f h}}{k_{f v}}} \\
\lambda=\frac{k_{m} L^{2}}{k_{f h} R^{2}} \\
\omega=\frac{\phi_{f} C_{t f}}{\phi_{f} C_{t f}+\phi_{m} C_{t m}} \\
r_{m D}=\frac{r}{R}
\end{gathered}
$$

\section{Appendix B}

Using the dimensionless variables (see Appendix A) for Eqs. (A-1)-(A-4), the following equations can be derived:

$$
\begin{gathered}
\frac{1}{r_{m D}^{2}} \frac{\partial}{\partial r_{m D}}\left(r_{m D}^{2} \frac{\partial p_{m D}}{\partial r_{m D}}\right)=\frac{(1-\omega)}{\lambda} \frac{\partial p_{m D}}{\partial t_{D}} \quad\left(0 \leq r_{m D} \leq 1\right) \\
P_{m D}\left(r_{m D}, 0\right)=0 \\
\left.\frac{\partial p_{m D}}{\partial r_{m D}}\right|_{r_{m D}=0}=0 \\
\left.p_{m D}\right|_{r_{m D}=1}=p_{f D}
\end{gathered}
$$

Taking Laplace transformation to $t_{D}$, Eqs. (B-1)-(B-4) becomes:

$$
\begin{gathered}
\frac{1}{r_{m D}^{2}} \frac{\partial}{\partial r_{m D}}\left(r_{m D}^{2} \frac{\partial \bar{p}_{m D}}{\partial r_{m D}}\right)=\frac{(1-\omega)}{\lambda} u \bar{p}_{m D} \quad\left(0 \leq r_{m D} \leq 1\right) \\
\bar{p}_{m D}\left(r_{m D}, 0\right)=0 \\
\left.\frac{\partial \bar{p}_{m D}}{\partial r_{m D}}\right|_{r_{m D}=0}=0 \\
\left.\bar{p}_{m D}\right|_{r_{m D}=1}=\bar{p}_{f D}
\end{gathered}
$$

Solve the Eqs. (B-5)-(B-8):

$$
\bar{p}_{m D}\left(r_{m D}, u\right)=\frac{\bar{p}_{f D}}{\sinh \sqrt{\frac{(1-\omega) u}{\lambda}}} \frac{\sinh \left[r_{m D} \sqrt{\frac{(1-\omega) u}{\lambda}}\right]}{r_{m D}}
$$


Using the dimensionless variables (see Appendix A), Eq. (A-5) can be derived:

$$
\frac{1}{r_{D}} \frac{\partial}{\partial r_{D}}\left(r_{D} \frac{\partial p_{f 1 D}}{\partial r_{D}}\right)+\frac{1}{r_{D}^{2}} \frac{\partial^{2} p_{f D}}{\partial \theta^{2}}+\frac{\partial^{2} p_{f D}}{\partial z_{D}^{2}}=\omega \frac{\partial p_{f D}}{\partial t_{D}}+\left.3 \lambda \frac{\partial p_{m}}{\partial r_{m D}}\right|_{r_{m D}=1}
$$

Taking Laplace transformation to $t_{D}$, Eq. (B-10) becomes:

$$
\frac{1}{r_{D}} \frac{\partial}{\partial r_{D}}\left(r_{D} \frac{\partial \bar{p}_{f D}}{\partial r_{D}}\right)+\frac{1}{r_{D}^{2}} \frac{\partial^{2} \bar{p}_{f D}}{\partial \theta^{2}}+\frac{\partial^{2} \bar{p}_{f D}}{\partial z_{D}^{2}}=\omega u \bar{p}_{f D}+\left.3 \lambda \frac{\partial \bar{p}_{m}}{\partial r_{m D}}\right|_{r_{m D}=1}
$$

With equation Eqs. (B-9)-(B-11), the flow equation in inner region can be derived:

$$
\begin{aligned}
& \frac{1}{r_{D}} \frac{\partial}{\partial r}\left(r_{D} \frac{\partial \bar{p}_{f D}}{\partial r_{D}}\right)+\frac{1}{r_{D}^{2}} \frac{\partial^{2} \bar{p}_{f D}}{\partial \theta^{2}}+\frac{\partial^{2} \bar{p}_{f D}}{\partial z_{D}^{2}}=f \bar{p}_{f D} \\
& f=\omega u+3 \lambda\left(\sqrt{\frac{1-\omega}{\lambda}} u \operatorname{coth} \sqrt{\frac{1-\omega}{\lambda}} u-1\right)
\end{aligned}
$$

